\title{
COMPUTATIONAL FLUID DYNAMICS PREDICTION OF THE RESISTANCE OF MODERN CONTAINER SHIPS
}

\author{
KIRIL TODOROV \\ Institute of Metal Science, Equipment and Technologies \\ with Hydro- and Aerodynamics Centre "Acad. Angel Balevski", \\ Bulgarian Academy of Sciences \\ 1, William Froude St., 9003 Varna, Bulgaria, \\ e-mail: k.todorov@bshc.bg
}

\begin{abstract}
The present paper is dedicated to the $45^{\text {th }}$ anniversary of the Bulgarian Ship Hydrodynamics Centre in Varna
\end{abstract}

\begin{abstract}
The present paper considers the capabilities of the open-source software for Computational fluid dynamics calculations Open Field Operation and Manipulation (OpenFOAM) to calculate the resistance of modern container ships. The calculations are performed for two container ships and the results are compared with model tests data.

Keywords: ship resistance, container ships, Computational fluid dynamics prediction.
\end{abstract}

\section{INTRODUCTION}

In recent years container shipping has increased significantly and this has led to an increase in the number of modern very large container vessels. These ships also travel at very high speeds for merchant ships. Because of the increase in the numbers of such vessels, two models of container ships were developed for academic purposes - Duisburg Test Case (DTC) and Korea Research Institute of Ships \& Ocean Engineering (KRISO) Container Ship (KCS). The DTC model was developed at Institute of Ship Technology, Ocean Engineering and Transport Systems (ISMT). The KCS model was developed at KRISO.

With the increase of computing power of modern central processing units, the capabilities of the software for Computational Fluid Dynamics (CFD) calculations have increased drastically.

In this paper the CFD prediction of the resistance of both container ships was done using the open-source software Open Field Operation and Manipulation (OpenFOAM).

DOI: 10.7546/EngSci.LVIII.21.03.04 


\section{OpenFOAM SOFTWARE}

The open-source software OpenFOAM was originally created as a commercial one, but was later changed to open-source. Since then, the software has been developed to its current form and now is as capable as most commercial software for CFD calculations.

Unlike most other softwares for CFD calculations OpenFOAM has not a Graphical user interface, but has a program one. This means the user must program the simulation using a text editor to create the files needed for every step of the process. Although this is difficult to learn and thus the software is not very user-friendly at the same time this design enables the user to program several additional simulations while running the CFD calculations. The software has its own moduli of creating the mesh (blockMesh and snappyHexMesh), refining the mesh and a large number of solvers depending on the scenario of the simulation. In OpenFOAM, the free surface is based on the Volume of Fluid (VOF) method. While OpenFOAM has not an integrated modulus of post-processing of the data, installing the software installs software called ParaView also, which is very capable post-processing software.

\section{GEOMETRY DESCRIPTION}

\subsection{Duisburg test case}

Duisburg test case is a 14000TEU post-Panamax container vessel. In this case the used scale is 1:59.407. The resistance tests for this ship are conducted in the model basin SVA Potsdam. In Figure 1, the hull shape of the DTC ship is shown. The main particvulars of the full scale ship and the used ship model are given in Table 1.

Table 1. Main particulars of DTC

\begin{tabular}{|c|c|c|}
\hline Main particulars & Model & Ship \\
\hline Lpp [m] & 5.976 & 355 \\
\hline $\mathrm{B}[\mathrm{m}]$ & 0.429 & 51 \\
\hline $\mathrm{T}[\mathrm{m}]$ & 0.244 & 14.5 \\
\hline $\mathrm{V}\left[\mathrm{m}^{3}\right]$ & 0.827 & 173467 \\
\hline $\mathrm{Cb}[-]$ & 0.661 & 0.661 \\
\hline $\mathrm{Sw}\left[\mathrm{m}^{2}\right]$ & 6.243 & 22032 \\
\hline
\end{tabular}

Engineering Sciences, LVIII, 2021, No. 3 

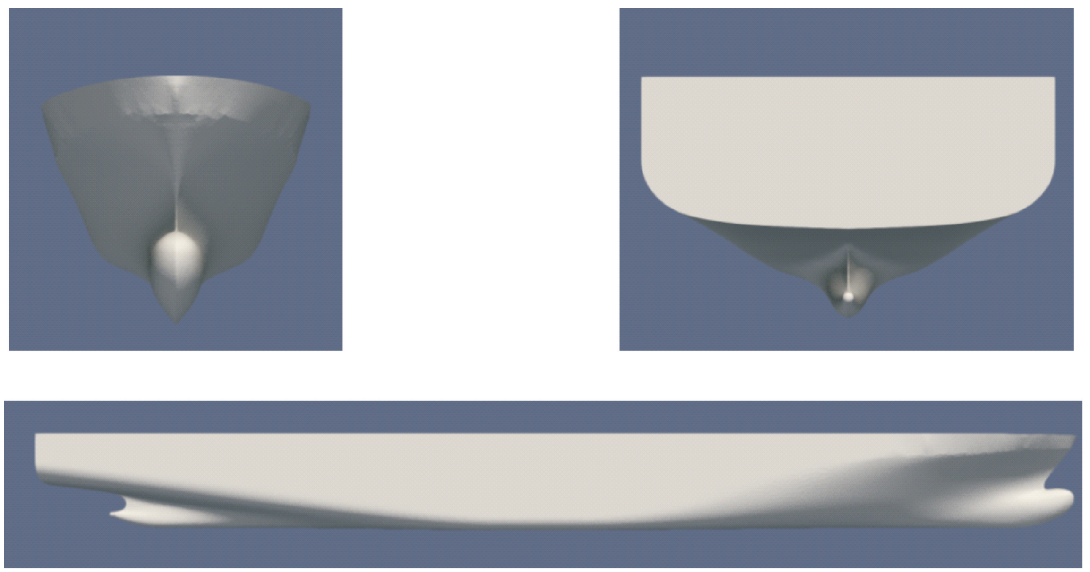

Fig. 1. Bow view, stern view and side view of DTC

\subsection{KRISO container ship}

As mentioned above the KCS is designed by KRISO. In this case the chosen model scale is $1 / 31.599$. In Figure 2, the hull shape of the KCS ship is shown. The main particulars of the full scale ship and the used ship model are given in Table 2.
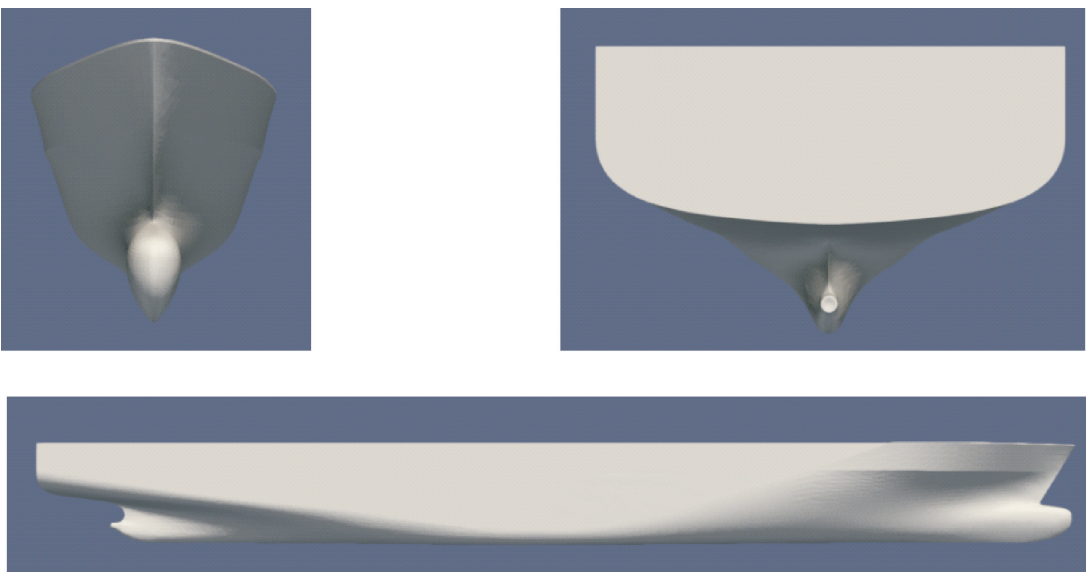

Fig. 2. Bow view, stern view and side view of KCS 
Table 2. Main particulars of KCS

\begin{tabular}{|c|c|c|}
\hline Main particulars & Model & Ship \\
\hline Lpp [m] & 7.2786 & 230 \\
\hline Lwl [m] & 7.3570 & 232.5 \\
\hline Bwl [m] & 1.0190 & 32.2 \\
\hline D [m] & 0.6013 & 19.0 \\
\hline T $[\mathrm{m}]$ & 0.3418 & 10.8 \\
\hline V $\left[\mathrm{m}^{3}\right]$ & 1.6490 & 52030 \\
\hline Sw $\left[\mathrm{m}^{2}\right]$ & 9.5441 & 9530 \\
\hline
\end{tabular}

\section{MESH STATISTICS AND COMPUTATIONAL SET-UP}

\subsection{Mesh statistics}

For both ships the domains were done following the International Towing Tank Conference requirements. The distance between the boundaries of the domains and the models are presented dimensionless, based on the overall length of the modes:

- X-axis: $2^{*} \mathrm{~L}_{\mathrm{OA}}$ ahead of the model and $4 * \mathrm{~L}_{\mathrm{OA}}$ behind the model;

- Y-axis: At least $2 * \mathrm{~L}_{\mathrm{OA}}$;

- Z-axis: At least $2^{*} \mathrm{~L}_{\mathrm{OA}}$ under the model and at least $1{ }^{*} \mathrm{~L}_{\mathrm{OA}}$ over the ship.

The procedure of generating the mesh is as follows:

- Firstly the domain is generated using blockMesh. In this step, the mesh around the free surface is also prepared. It should be noted that the mesh in the free surface region must be finer than the rest of the domain. In this way, the waves generated by the ships can be captured much more accurately.

- The second step is to run refineMesh which refines the mesh. The application is run a number of times for different regions of the domain, starting from the biggest and going towards the smallest.

- After the refinement is finished the next step is to run snappyHexMesh. This application snaps the mesh around the 3D model, which has been selected for the case. This application not just snaps the mesh around the model, but also refines it and creates the boundary layers around the hull so that the turbulent flow can be correctly simulated. 


\subsubsection{Mesh details for Duisburg test case}

For Duisburg test case the free surface region is between $0.188 \mathrm{~m}$ and 0.3 $\mathrm{m}$ on the Z-axis. The number of surface layers is set to 3 . The minimum $\mathrm{y}+$ value is 1.53 and the maximum is 703.72 , which gives an average value of 65.22. The refinement of the mesh is done in 6 stages. In Table 3 , the cell types and their numbers are given, and Fig. 3 shows the mesh.
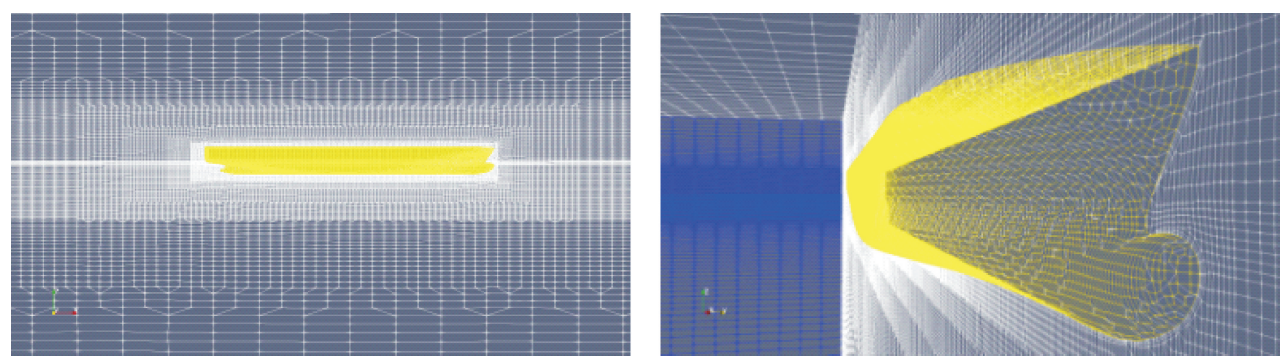

Fig. 3. Mesh around the hull of DTC

Table 3. Mesh stats for DTC

\begin{tabular}{|c|c|}
\hline Mesh stats & Total count \\
\hline Cells & 851196 \\
\hline Hexahedral cells & 794450 \\
\hline Polyhedral cells & 55281 \\
\hline
\end{tabular}

\subsubsection{Mesh details for KRISO container ship case}

For KRISO container ship case the free surface region is between $0.244 \mathrm{~m}$ and $0.4 \mathrm{~m}$ on the Z-axis. The refinement is done in 5 stages. The number of surface layers is set to 5 . The minimum $\mathrm{y}+$ value is 0.6 and the maximum is 2321.13, which gives an average value of 48.95. In Table 4, the cell types and their numbers are given. Figure 4 shows the mesh.
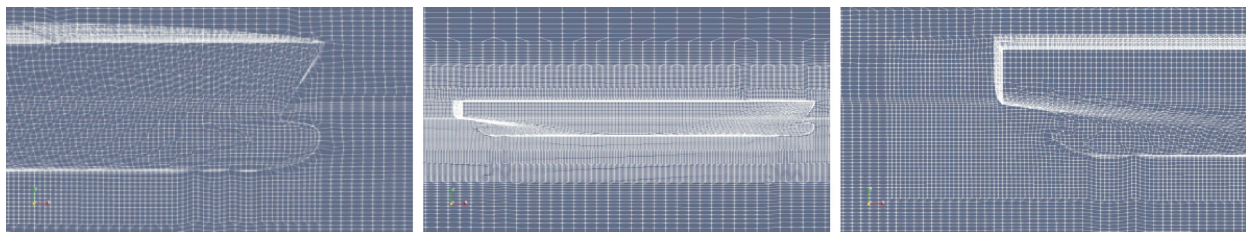

Fig. 4. Mesh around the hull of KCS 
Table 4. Mesh stats for KCS

\begin{tabular}{|c|c|}
\hline Mesh stats & Total count \\
\hline Cells & 605120 \\
\hline Hexahedral cells & 564060 \\
\hline Polyhedral cells & 40425 \\
\hline
\end{tabular}

\subsection{Computational set-up}

For both the DTC and KCS the computational set-up is the same. The used solver is interFoam, which is for multiphase fluids based on the ReynoldsAveraged Navier-Stokes (RANS) method. The chosen turbulence model is $k-$ $\omega$ Shear Stress Transport (SST), which is a turbulent model for incompressible flows.

For the pressure the solver Generalized Geometric-Algebraic Multi-Grid with smoother GaussSeidel is used, the tolerance is set to $1^{*} \mathrm{e}^{-8}$. For $U, k$ and $\omega$ the used solver is smoothSolver with smother symGaussSeidel, the tolerance is set to $1^{*} \mathrm{e}^{-7}$.

The divergence schemes were chosen as follows:

- For $U$ - Gauss linearUpwind grad (U);

- For $k$-Gauss linearUpwind limitedGrad;

- For $\omega$ - Gauss linearUpwind limitedGrad.

\section{NUMERICAL RESULTS}

In order to make a comparison between the model tests and CFD calculations, the resistance is used to calculate coefficient of total resistance $C_{T}$ and frictional resistance coefficient $C_{F}$. The used formulae are:

$$
\begin{aligned}
& C_{T}=R_{T} /\left(0.5 \rho S w V^{2}\right), \\
& C_{F}=R_{F} /\left(0.5 \rho S w V^{2}\right) .
\end{aligned}
$$

The results for the two coefficients are multiplied by $10^{3}$ because the values of the coefficients are too small numbers, presented in two ways: in tabular form and as a diagram.

Engineering Sciences, LVIII, 2021, No. 3 


\subsection{Results for Duisburg Test Case}

The results from the simulations using the DTC hull model are compared to the results published in [1]. The frictional coefficient $C_{F}$ in the Experimental Fluid Dynamics (EFD) cases has been calculated using RANS-CFD code [1]. The results from both the EFD and CFD are compared in Table 5 and Fig. 5.

Table 5. Coefficient of total resistance for DTC as the Froude number increases

\begin{tabular}{|c|c|c|c|c|c|c|}
\hline Fr & OF & EFD & Error, $\%$ & OF & EFD & Error, $\%$ \\
\hline & $\mathrm{C}_{T}^{*} 10^{\wedge} 3$ & $\mathrm{C}_{T}{ }^{*} 10^{\wedge} 3$ & & $\mathrm{C}_{F}{ }^{*} 10^{\wedge} 3$ & $\mathrm{C}_{F}{ }^{*} 10^{\wedge} 3$ & \\
\hline 0.174 & 3.4710 & 3.661 & -5.466 & 2.927 & 3.170 & -7.676 \\
\hline 0.183 & 3.5350 & 3.605 & -1.980 & 2.990 & 3.142 & -4.846 \\
\hline 0.192 & 3.5124 & 3.588 & -2.164 & 2.970 & 3.116 & -4.685 \\
\hline 0.200 & 3.4430 & 3.602 & -4.624 & 2.930 & 3.092 & -5.228 \\
\hline 0.209 & 3.4610 & 3.623 & -4.683 & 2.914 & 3.069 & -5.048 \\
\hline 0.218 & 3.5810 & 3.670 & -2.476 & 2.913 & 3.047 & -4.409 \\
\hline
\end{tabular}

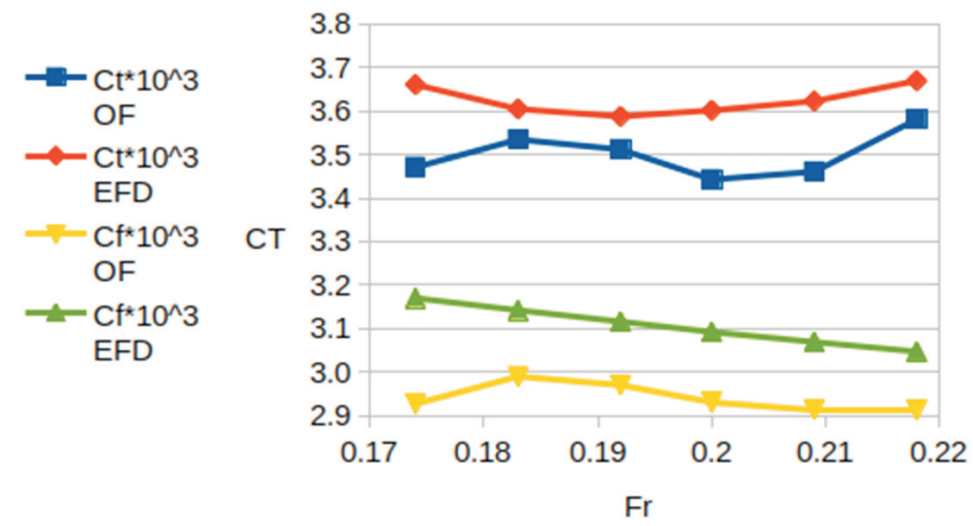

Fig. 5. Chart representation of the total resistance coefficient for model tests and CFD

The results show a marginal error percentage for the coarse mesh in this case. A grid dependence analysis should be performed in the future. 


\subsection{Results for KRISO container ship}

In the case of KRISO container ship, a comparison is made not only between the results of model tests and CFD calculations with OpenFOAM, but also with the NUMECA FINE ${ }^{\mathrm{TM}} /$ MARINE software, Table 6 and Fig. 6.

Table 6. Coefficient of total resistance for KCS as the Froude number increases

\begin{tabular}{|c|c|c|c|c|c|}
\hline Fr & EFD & FINE/MARINE & Error, \% & OF & Error, \% \\
\hline 0.1083 & 3.80 & 3.73 & -1.877 & 3.916 & 2.964 \\
\hline 0.1516 & 3.64 & 3.69 & -1.111 & 3.667 & 0.741 \\
\hline 0.1949 & 3.48 & 3.46 & -0.578 & 3.527 & 1.339 \\
\hline 0.2274 & 3.47 & 3.49 & 0.573 & 3.518 & 1.351 \\
\hline 0.2599 & 3.71 & 3.70 & -0.270 & 3.668 & -1.152 \\
\hline 0.2816 & 4.50 & 4.52 & 0.442 & 4.338 & -3.732 \\
\hline
\end{tabular}

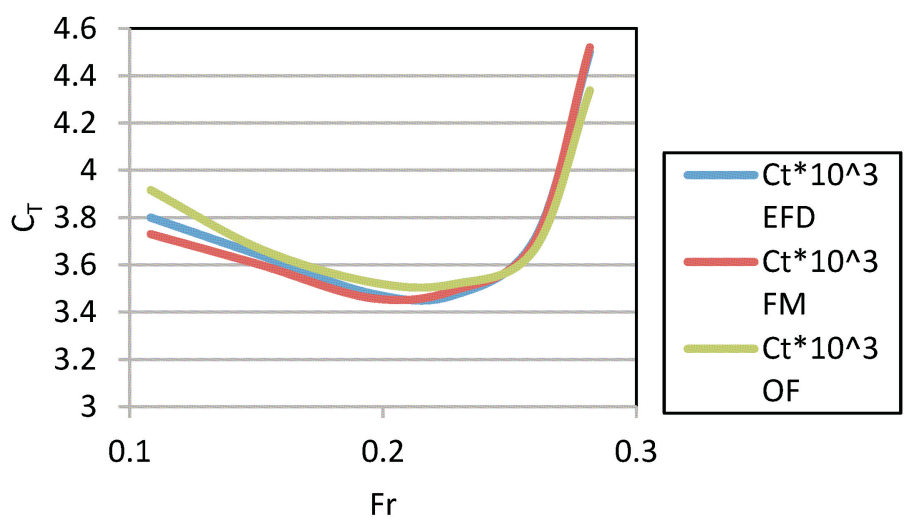

Fig. 6. Chart representation of the total resistance coefficient for model tests and CFD

The results for the KCS model are much better than the ones for the DTC model. This could be a result of the different mesh structures, which would be further analyzed. Despite the coarser mesh the error percentage is much lower and the curve is almost the same as the ones generated by EFD experiments and NUMECA FINE ${ }^{\mathrm{TM}} /$ MARINE.

Engineering Sciences, LVIII, 2021, No. 3 


\section{3D GRAPHIC RESULTS OF THE SIMULATIONS}

When doing CFD calculations, part of the post-processing process is a graphical representation of the results of these calculations. Graphical results taken from the simulations of the cruise speed of both ships are presented here.

\subsection{Duisburg test case}

Several views of the free surface of the model and the pressure distribution around the hull are shown in Figs 7-9.

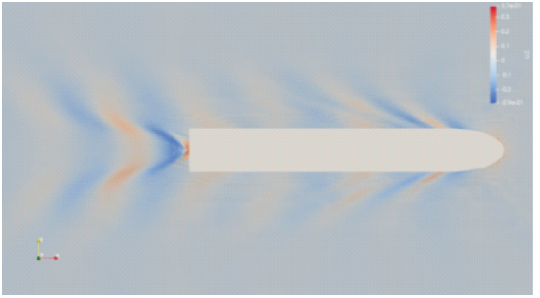

(a)

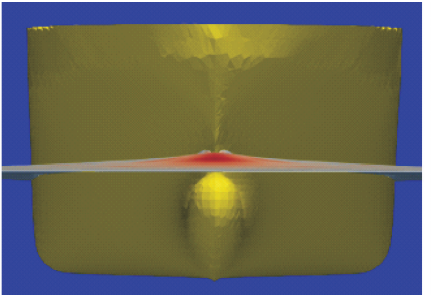

(b)

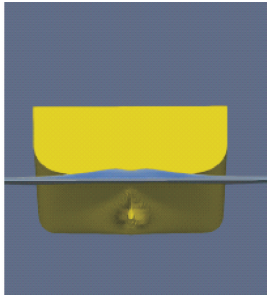

(c)

Fig. 7. (a) Top view of the free surface; (b) free surface seen from the bow; (c) stern view of the free surface

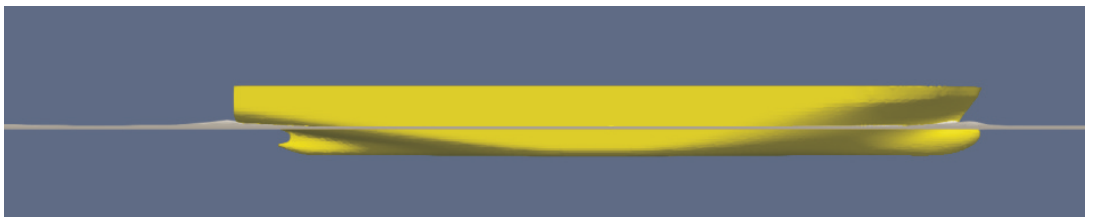

Fig. 8. Side view of the free surface

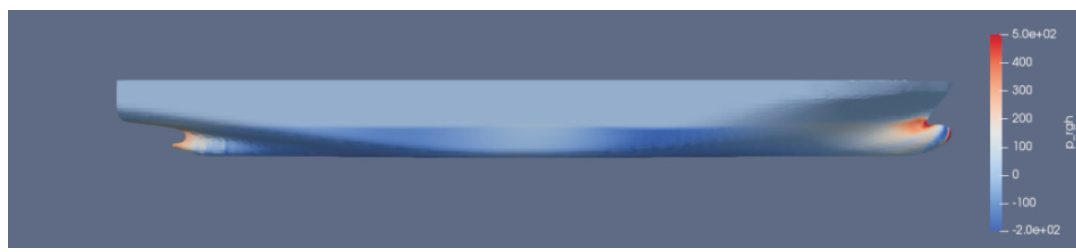

Fig. 9. Pressure distribution over the hull of DTC for Fr. 0.218 


\subsection{KRISO container ship}

Several views of the free surface, pressure distribution around the hull and stream lines around the hull are shown in Figs 10-13.

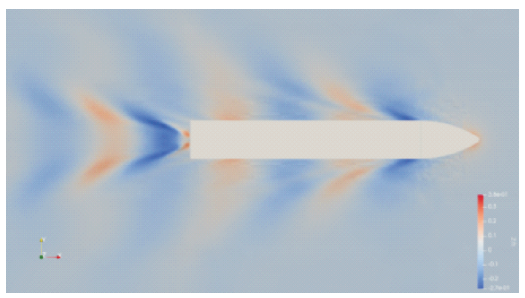

(a)

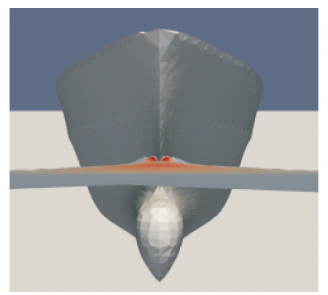

(b)

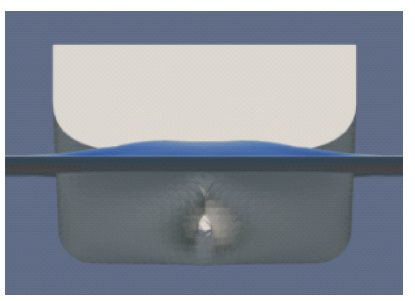

(c)

Fig. 10. (a) Top view of the free surface; (b) free surface seen from the bow; (c) stern view of the free surface
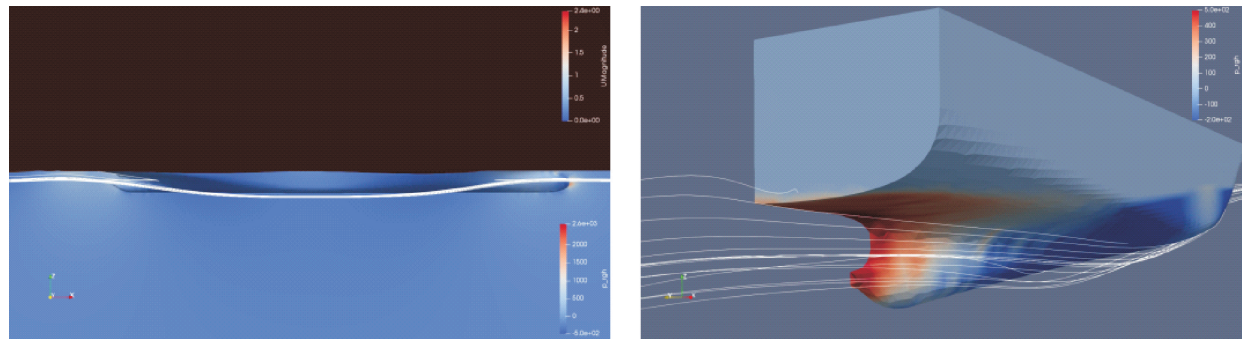

Fig. 11. Streamlines under the free surface for Fr. 0.2599

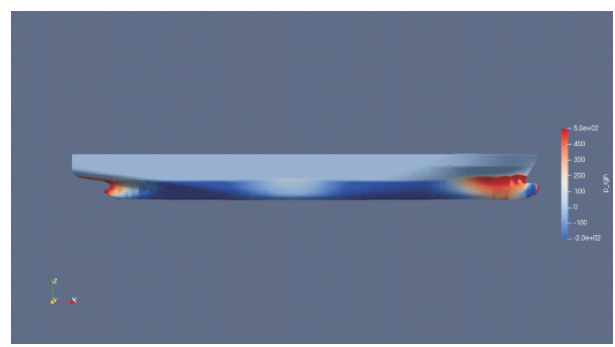

Fig. 12. Pressure distribution on KCS model for Fr. 0.2599

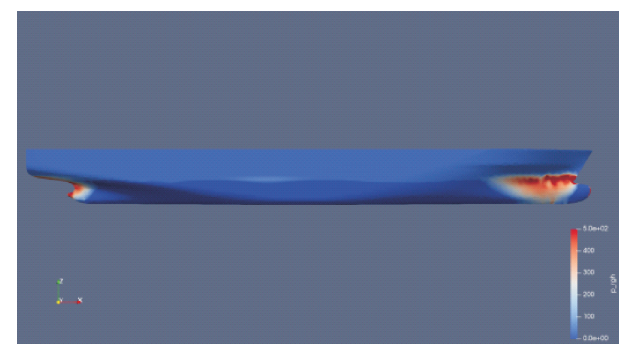

Fig. 13. Pressure distribution on KCS model for Fr. 0.2816 


\section{CONCLUSION}

Although these two cases are run with very coarse meshes, the results calculated by means of OpenFOAM have been very accurate. This shows that OpenFOAM is very capable software for CFD calculations and has a good future use in the field of ship hydrodynamics. The results of the CFD calculations show that both ships have similar resistance, i.e. the hull form of modern high-speed container is optimized to a very high level.

\section{REFERENCES}

[1] Ould el Moctar, Vladimir Shigunov, and Tobias Zorn, Duisburg Test Case: Post-Panamax Container Ship for Benchmarking, Ship Technology Research (2012) 59 (3) 50-64.

[2] Ludwig Kerner, KCS Resistance Calculation, Benchmark Report_2014-0919_FM_Resistance-KCS, https://www.numeca.com/docs/finemarine_kcs_g2010_21-2-3_18092014.pdf.

Received April 19, 2021 